Linguistique, littérature, didactique

$157-158 \mid 2013$

Théories et pratiques des genres

\title{
La dissertation : un genre scolaire argumentatif ?
}

Perspective historique

Nathalie Denizot

\section{(2) OpenEdition}

Journals

Édition électronique

URL : http://journals.openedition.org/pratiques/3823

DOI : $10.4000 /$ pratiques.3823

ISSN : 2425-2042

Éditeur

Centre de recherche sur les médiations (CREM)

Édition imprimée

Date de publication : 1 juin 2013

Pagination : 165-176

Référence électronique

Nathalie Denizot, «La dissertation : un genre scolaire argumentatif ? », Pratiques [En ligne],

157-158 | 2013, mis en ligne le 18 décembre 2017, consulté le 19 avril 2019. URL : http://

journals.openedition.org/pratiques/3823; DOI : 10.4000/pratiques.3823

(c) Tous droits réservés 


\title{
La dissertation : un genre scolaire argumentatif ? Perspective historique
}

\author{
Nathalie Denizot \\ Université de Cergy-Pontoise - IUFM, EMA (ÉA 4507)
}

Dans les textes officiels des années 2000-2010, la dissertation est clairement liée à l'argumentation, comme le montre notamment la définition actuelle de l'épreuve à l'EAF (Épreuves anticipées de français ; $B O$ spécial $\mathrm{n}^{\circ} 7 \mathrm{du} 6$ octobre 2011), qui est restée strictement identique depuis 2001 :

La dissertation consiste à conduire une réflexion personnelle et argumentée à partir d'une problématique littéraire issue du programme de français. Pour développer son argumentation, le candidat s'appuie sur les textes dont il dispose, sur les « objets d'étude » de la classe de première ainsi que sur ses lectures et sa culture personnelle.

Cette définition fait écho à de nombreux passages des programmes : les lycéens « doivent être capables, à partir de leurs lectures, de formuler un jugement personnel argumenté, notamment dans un commentaire ou une dissertation »; « les exercices d'analyse, de commentaire et de dissertation concourent » à « la maîtrise de l'expression écrite autonome » dans " les écrits d'argumentation et de délibération » (programme de première, $B O \mathrm{n}^{\circ} 28 \mathrm{du} 12$ juillet 2001) ; et dans le programme de seconde comme dans celui de première, commentaire et dissertation sont les deux seuls exercices cités dans la rubrique de « l'écriture d'argumentation » (BO spécial 9 du 30 septembre 2010).

La dissertation est donc enrôlée du côté de l'argumentation, et apparaît ainsi comme un « genre argumentatif». C'est cette évidence — partagée d'ailleurs en partie par d'autres disciplines la pratiquant (Denizot et Mabilon-Bonfils, 2012) que je voudrais ici interroger : en effet, son apprentissage est, dans les manuels actuels destinés aux classes des lycées, déconnecté des genres argumentatifs au programme, et en réalité peu tourné vers l'argumentation. J'explorerai ce paradoxe à partir d'un retour sur l'histoire de l'exercice (principalement à travers les textes officiels et les ouvrages méthodologiques de différentes époques), pour mieux comprendre comment le genre s'est « sédimenté » (Schneuwly, 2007) et plusieurs fois reconfiguré, et pour mieux en saisir les enjeux — passés et présents. Chemin faisant, c'est le fonctionnement de la dissertation en tant que genre scolaire que je tenterai ainsi d'éclairer. 


\section{Les fondements historiques du genre scolaire « dissertation »}

Née d'une certaine manière contre la rhétorique et surtout comme une alternative au discours, exercice-roi des classes de rhétorique (Chervel, 2006, p. 668 sq.), la dissertation s'impose progressivement à partir de 1880 . Sans revenir ici sur une histoire bien documentée (voir notamment Chervel, 2006 ; Jey, 1998 ; HoudartMérot, 1998), je voudrais souligner deux points qui me paraissent essentiels pour comprendre les fondements du genre, ses composantes historiques.

\section{La dissertation comme mise en scène de savoirs}

Au début du XX ${ }^{\mathrm{e}}$ siècle, la dissertation s'ancre dans la discipline en articulation avec l'explication de textes, devenue à partir de 1902 l'exercice principal de l'enseignement secondaire : comme le souligne Chervel (id., p. 688) : «c'est sur [1'explication de textes] que l'on compte pour faire de la dissertation littéraire la clé de voûte du nouvel enseignement du français ». En l'absence de «commentaire » (l'exercice n'apparaîtra au baccalauréat qu'en 1969), de nombreux sujets de dissertations sont des sujets d'analyse littéraire, qui s'apparentent en réalité à des explications de textes rédigées. Les corrigés des annales de ces époques en témoignent : sur un sujet classique tel que « Vous étudierez, en une dissertation suivie, l'inspiration et l'art de Baudelaire dans ce poème [Spleen] » $\left(1^{\mathrm{re}}\right.$ session de 1959), le corrigé des annales Bordas, par exemple (Juneaux, 1960), propose une sorte d'explication de textes rédigée et composée (en deux parties), qui mêle réflexions générales sur Baudelaire et le spleen, et analyse du poème. Certains traités méthodologiques envisagent d'ailleurs les deux exercices dans un même ouvrage : c'est ainsi par exemple que, dans la cinquième édition de son ouvrage à destination des élèves, Joseph Toinet (1947) ajoute aux « dissertations littéraires » des explications de textes, s'en expliquant ainsi dans sa préface (p. 6) :

J'ai ajouté à mes Dissertations littéraires, pour cette cinquième édition, vingt-cinq explications de textes. L'économie générale du livre n'en est pas modifiée : chacune de ces explications est insérée à son rang chronologique ou à sa place logique. Pas davantage la méthode et les principes suivis pour les plans de dissertation ne se trouveront contredits. L'explication française [...] est un commentaire développé, où l'on garde évidemment ses coudées plus franches, mais toujours organisé, de même que la dissertation, selon un plan directeur.

Les solidarités entre les deux exercices sont ici clairement exprimées, et les explications de textes ne sont pas répertoriées séparément dans l'ouvrage, mais sont intercalées au milieu des sujets de dissertation, où elles sont traitées de la même manière, en quelques points qui mêlent considérations sur les œuvres et les auteurs et considérations d'histoire littéraire.

Au milieu du $\mathrm{XX}^{\mathrm{e}}$ siècle, la dissertation permet donc, tout comme l'explication de textes, d'évaluer des savoirs d'histoire littéraire, d'autant que les « sujets généraux » ont tendance à devenir l'apanage des dissertations de philosophie ${ }^{(1)}$. Les ouvrages méthodologiques mettent l'accent sur les contenus : des Granges et Maguelonne (1929) consacrent un chapitre à « ce qu'il faut savoir de l'histoire littéraire "; Bénac (1949) publie un Vocabulaire de la dissertation qui propose une

(1) C'est déjà le constat que fait Mornet en 1934: "Ces sujets généraux tendent à disparaître du baccalauréat de la classe de première, car on discute plus efficacement les problèmes de morale, de psychologie, d'esthétique dans la classe de philosophie. » (p. 51) 
liste alphabétique de notions essentiellement littéraires ; Clouard (1961) réduit la partie méthodologique à la portion congrue (une double page) et consacre tout le reste (près de 600 pages) à des analyses « qui fournissent exactement ce qu'il y a d'essentiel à savoir d'un auteur ou d'un ouvrage en vue de l'examen » (p. X). La plupart des traités proposent aux élèves un grand nombre de dissertations corrigées, les sujets ayant été « choisis avec soin parmi ceux qui, depuis vingt-cinq ans, "sortent" le plus souvent » (Thoraval, 1959, p. 7). Puisque la dissertation est essentiellement le lieu de l'évaluation de savoirs littéraires, les ouvrages méthodologiques sont conçus comme une aide au bachotage. Comme 1'écrit Clouard dans l'avant-propos de son manuel (1961, p. IX) : « Tous les sujets de composition littéraire $[\ldots]$ se ramènent à un certain nombre de connaissances capitales. Eh bien, ces connaissances se trouvent rassemblées ici [...]. » De ce point de vue, la dissertation participe des genres évaluatifs propres à l'école : il faut savoir et surtout montrer que l'on sait. Et cette fonction de mise en scène de savoirs, essentielle dans la construction du genre, reste une dimension importante dans sa configuration actuelle : la dissertation est proche de certains exercices comme certaines formes d'exposé ou comme la « question de synthèse » qui a existé à certaines époques à l'oral de l'EAF — deux genres scolaires qui ne sont pas argumentatifs.

\section{Une « composition française »}

À la fin du XIX ${ }^{\mathrm{e}}$ siècle, la dissertation est l'une des formes que peut prendre la «composition française » ${ }^{(2)}$. Or, cette dénomination générique, qui subsiste jusque dans les années 1970 (et qui reviendra d'ailleurs entre 1983 et 1994 : cf. infra) signale bien l'importance des aspects compositionnels. Les ouvrages méthodologiques n'ont de cesse de le rappeler, et je prendrai un exemple (Thoraval, 1959, p. 6) parmi tant d'autres possibles :

D'une session à l'autre, les correcteurs signalent les mêmes fautes essentielles. Ils se plaignent moins encore du manque de connaissances que de la faiblesse de la mise en œuvre : du désordre du plan, des imprécisions dans le détail, de la sécheresse et des impropriétés du style.

Dissertation et explication de textes partagent d'ailleurs cette même obsession du «plan » et de l'enchaînement des idées : comme le souligne Martine Jey (1998, p. 91), « expliquer [un texte], c'est retrouver un ordre ». C'est ainsi que Mornet (1934, p. 69) associe plan d'explication et plan de dissertation dans son ouvrage sur la composition française :

Il est utile d'habituer les élèves à faire avec exactitude le plan d'un texte. C'est un excellent exercice pour les amener à composer eux-mêmes avec ordre un plan de dissertation : ils apprendront à ne pas s'égarer dans les détails, mais à ordonner avec clarté la matière d'un développement.

La dissertation se caractérise donc au moins autant par sa composition que par son contenu, et de ce point de vue, elle prolonge les enseignements de la rhétorique, envisagée comme technique de production de textes : analysant un grand nombre de copies entre 1880 et 1960, Violaine Houdart-Mérot (1998) a bien montré la «persistance de la tradition rhétorique» (p. 69), tant dans les sujets que dans leur traitement par les élèves (amplification de la pensée de l'auteur, manière d'é-

(2) Narrations, lettres, discours, etc. tiennent une place non négligeable jusque dans les années 1960 . 
crire) et dans les annotations des correcteurs, qui « continuent [après 1925] à porter prioritairement sur l'organisation d'ensemble du devoir » (p. 113). Les approches traditionnelles de la dissertation la rapprochent ainsi des anciens exercices rhétoriques, et l'on retrouve là les mécanismes bien connus de recyclage des pratiques d'enseignement.

Cette composition impose d'organiser des exemples et des « arguments », mais les termes sont à prendre ici au sens rhétorique, dans la tradition des « lieux communs ». Comme le rappelle Vigner (1990, p. 22) : "L'élève n'a pas à faire preuve d'originalité mais doit s'inscrire dans un espace intertextuel qui garantit l'intelligibilité du propos. » C'est dans ce contexte qu'il faut replacer par exemple cette définition de l'exercice chez Bénac (1949, p. VI) :

\begin{abstract}
Dissertation ou Composition française au baccalauréat.
Exercice scolaire qui consiste tantôt à étudier un texte pour montrer l'intérêt de ses idées ou l'agrément de son style, tantôt à traiter, à partir d'un jugement ou d'une question, un problème de littérature ou d'idées. Dans tous les cas, le sujet de la composition doit être posé dans une entrée en matière et divisé en plusieurs points. Chaque point est développé dans une partie distincte à l'aide d'arguments nettement classés et appuyés sur des preuves fournies par des faits ou des exemples tirés des œuvres. Le développement aboutit à une conclusion qui, après avoir résumé les arguments, en dégage le sens et donne une solution au problème posé dans l'entrée en matière.
\end{abstract}

La description de l'exercice renvoie ici nettement aux anciennes techniques de la rhétorique et à son formalisme textuel. Or, si rhétorique et argumentation ont partie liée, les deux ne sont pourtant pas équivalentes : comme le souligne Claudine Garcia-Debanc (2001, p. 194), la tradition rhétorique « propose une approche prescriptive, normative, édictant les règles de réussite de chacun des genres de discours » et « s'intéresse essentiellement à la production de discours ", alors que l'argumentation (telle que l'envisagent les approches actuelles) « se veut plutôt descriptive, analytique et unificatrice ", "s'intéresse plus souvent à la réception des discours qu'à leur production et se garde d'édicter la moindre instruction». Tel qu'il est envisagé jusqu'aux années 1960, le genre scolaire de la dissertation est donc davantage tourné vers la rhétorique que vers l'argumentation, avec de fortes composantes compositionnelles.

\title{
Deux tentatives de reconfiguration de la dissertation
}

Au tournant des années 1960, la dissertation est donc un genre composite : elle emprunte aux anciens genres rhétoriques scolaires (discours, narrations) des modèles formels et des techniques (dispositio, inventio); elle emprunte à l'explication de textes des contenus et des discours sur les textes, voire un rapport aux textes, fondé sur l'admiration et la célébration. Mais le genre s'essouffle, et face aux difficultés de l'exercice, le Mémento à l'usage des professeurs et élèves-professeurs de Lettres et Grammaire, publié sous l'égide du Ministère de l'Éducation Nationale par l'Institut Pédagogique National en 1953 (et réédité jusqu'en 1964), en arrive même à conseiller aux enseignants d'utiliser un " canevas », un «plan [qui] indiquera d'avance les principaux points à traiter » et qui « évite de demander aux élèves plus qu'ils ne peuvent et les garde du découragement comme de la présomption ». C'était relégitimer les pratiques rhétoriques de l'amplification à partir d'une « matière " — pratiques que l'instauration de la composition française avait justement voulu éradiquer. Dans une forme d'impasse, la dissertation va pourtant trouver un second 
souffle à travers deux tentatives de reconfiguration : sa transformation en « essai » tout d'abord, puis surtout son rattachement à l'argumentation.

\section{Quand la dissertation devient essai}

Le début des années 1970 marque un moment important dans l'histoire de la dissertation. La création de l'EAF en 1969 est en effet corrélée à de profondes modifications des épreuves du baccalauréat en français ; la composition française disparaît $^{(3)}$ et de nouveaux exercices apparaissent, dont l' "essai littéraire », ainsi défini par la circulaire de $1972^{(4)}$ :

Le sujet pose, éventuellement à partir d'une citation, une question largement ouverte, qui n'appelle pas une réponse unique et prédéterminée. Cette question est formulée de manière que chaque candidat se sente capable de la résoudre pour son propre compte, selon ses propres vues, en tirant parti à sa convenance des lectures qu'il a faites en classe ou hors de la classe et sur lesquelles il peut fonder un jugement motivé. Il ne s'agit donc ni d'une « question de cours » portant sur un ouvrage, un auteur, un mouvement impérativement désigné, ni d'un débat de doctrine, portant sur la définition théorique d'un genre, la genèse ou les effets généraux des productions de la littérature. L'intérêt des réflexions ainsi formées et des illustrations ou des justifications qui les éclairent est mis en valeur par la solidité de la composition.

Cette définition, reconduite dans les diverses circulaires jusqu'en 1983 (date à laquelle 1'épreuve est à nouveau redéfinie : $c f$. infra) est intéressante à plusieurs titres : tout d'abord parce qu'elle dessine en creux l'ancien exercice de dissertation, à qui l'on reproche d'être devenu une «question de cours » ou un « débat de doctrine »; ensuite parce qu'elle évoque ce qui fait de cette nouvelle épreuve un " essai » («résoudre pour son propre compte » etc.); enfin par ce qu'elle révèle de l'attachement malgré tout de ce nouvel exercice à la tradition scolaire, qui doit toujours se caractériser par " la solidité de la composition».

Or, rebaptiser la dissertation « essai » marque une rupture avec le modèle traditionnel de la dissertation sur plusieurs points essentiels. Sur le plan compositionnel, on pouvait tout d'abord imaginer des formes textuelles plus souples, puisque c'est cette souplesse voire cette façon de défier les normes génériques qui est souvent considérée comme le propre du genre (Glaudes et Louette, 1999). Sur le plan énonciatif, on pouvait également envisager une rupture avec les contraintes de la dissertation (notamment l'interdit du « je »), l'essai se caractérisant justement dans la tradition littéraire française (de Montaigne à Gracq en passant par Rousseau ou Chateaubriand : voir Macé, 2006) par la présence d'un «je » qui sans être autobiographique ni intimiste s'affiche et s'affirme clairement. Enfin, la définition de l'exercice qui met en avant le jugement personnel de l'élève, voire ses lectures « hors de la classe», fait écho aux nouvelles conceptions de la lecture qui se font en effet jour à partir des années 1960 (Chartier et Hébrard, 2000) : il ne s'agit plus d'admirer inconditionnellement, mais de laisser place à la « culture personnelle » des élèves et à leurs lectures privées (Veck, 1994). La première définition de l'essai en 1969 évoque même les « réactions authentiques que les candidats ont

(3) Cela n'empêche pas l'expression de subsister encore, comme en témoigne par exemple le titre des annales Vuibert des années 1970: "Compositions françaises $1^{\text {re }}$ et Terminales ».

(4) Circulaire $n^{\circ} 72-455$ du 23 novembre 1972. Une première définition de l'épreuve avait été publiée en 1969, mais rapidement revue pour laisser place à celle-ci, qui restera stable jusqu'en 1983. 
éprouvées au contact de pages dont ils ont gardé un vif souvenir et sur lesquelles ils sont invités à motiver à leur gré un sentiment sincère, révélateur de leur personnalité, de leur culture, de leur jugement, de leur goût ». L'essai veut ainsi rompre avec ce qui apparaît alors comme les limites de la dissertation, devenue comme je 1'ai rappelé un exercice de bachotage et de composition. Loin des canevas et des amplifications, "l'essai suppose le souci de former des consciences critiques, spécialistes de l'analyse et de la synthèse ", comme l'écrivent par exemple Biet et al. (1988, p. 205).

Qu'en est-il en réalité ? Dans les manuels ${ }^{(5)}$ et les sujets de cette époque, l'essai ne se distingue pas de la dissertation - le mot n'a d'ailleurs pas disparu et certains (Brunel et Couty, 1977 par exemple) n'utilisent que lui. Rincé (1980) propose à la fois des sujets de « dissertation » et des sujets d' « essai », et si certains sujets d'essai sont proches de ce qu'on appelle maintenant l'écriture d'invention («Vous rédigerez vous-même une description "aérienne" du Paris moderne ou de toute autre grande ville », p. 68), d'autres sont explicitement des dissertations (« Essai. Vous discuterez sous forme de dissertation cette analyse de Baudelaire... », p. 70). Cette relative souplesse de l'essai, dans ce manuel du moins, ne se retrouve en rien dans les annales, qui offrent des sujets très proches de ceux que l'on pouvait trouver auparavant. Il suffit de comparer ces deux énoncés, à vingt ans d'écart, pour se rendre compte que la différence tient davantage à la forme de l'énoncé lui-même (plus développé et explicite en 1980) qu'à la question posée et au genre attendu :

- Montrez en faisant appel à votre expérience personnelle ce que la représentation d'une pièce ajoute à sa lecture ( $2^{\mathrm{e}}$ session, 1959, dans Juneaux, 1959, p. 5)

- Beaucoup affirment que le théâtre ne prend son véritable sens que pendant la représentation et grâce à elle : la simple lecture de l'œuvre rédigée par l'écrivain passerait ainsi à côté de l'essentiel : on ne peut pas «lire » le théâtre.

Vous tenterez de dire en vous référant à des textes de théâtre étudiés en classe ou à d'autres expériences de spectacle, ce que vous pensez de cette affirmation. (Lille. EAF 1979: A, B, C, D, E, dans Géray, 1981, p. 167)

Rien de surprenant finalement si l'essai littéraire, dès 1983, disparaît entièrement au profit de la dissertation, redevenue « composition française sur un sujet général » : la tradition scolaire l'a emporté. D'ailleurs, la contrainte, dès 1972, d'un essai « composé » (ce qui signifie en réalité organisé selon le modèle scolaire de l'introduction/développement/conclusion) soulignait bien l'importance du niveau compositionnel et portait en germe la soumission de l'essai aux normes scolaires. L'ancien modèle de la dissertation a pesé sur l'essai, qui n'a pas eu le temps de s'en affranchir. Un des rares manuels de méthodes de cette époque (Pagès et Pagès-Pindon, 1982) prend ainsi acte de ce qui n'est qu'un changement de nom : la réédition de 1984 se contente de remplacer « essai littéraire » par " composition française » dans le chapitre la concernant. Pour le reste, aucun mot n'est changé. L'essai n'avait jamais été rien d'autre qu'un avatar à peine modernisé de la dissertation.

\section{Dissertation et discussion}

La force de la tradition scolaire a joué un rôle important dans l'alignement de l'essai sur la dissertation, et dans ce qui peut être lu comme l'échec d'une tentative

(5) Les manuels de méthode sont encore très peu nombreux au tout début des années 1980. J'ai donc regardé aussi les sujets proposés dans les manuels de textes ainsi que dans diverses annales. 
de renouvellement de l'exercice. Un autre facteur me semble cependant déterminant : l'émergence de l'argumentation dans les textes officiels au cours des décennies 1980-1990 (Benoit, 1999), qui va venir reconfigurer le genre de la dissertation aux dépens de l'essai. L'argumentation ${ }^{(6)}$, tout d'abord objet de travail ancillaire, s'articule aux nouveaux objectifs des programmes scolaires (apprendre à communiquer; acquérir des techniques et des méthodes pour viser l'autonomie de l'élève) et à de nouveaux exercices, notamment la « discussion ». Cette dernière, qui subsistera sous diverses formes jusqu'en 2003, n'est pas isolée dans ces années 1970-1980. On la retrouve sous des formes diverses en BEP, en CAP puis à partir de 1987 au brevet des collèges : arrivent ainsi des sujets d'examen proposant des écrits plus courts et moins codés sans doute que l'ancienne dissertation, mettant en avant l'enjeu argumentatif (« discussion »), et non plus seulement la forme textuelle («composition») ${ }^{(7)}$ ni les contenus (la discussion pouvant porter sur n'importe quel sujet).

La discussion est donc rapidement incontournable, et entre en concurrence avec l'essai. Or, dans les ouvrages méthodologiques pour le lycée, elle est présentée comme une «petite dissertation » (Biet et al., 1988, p. 168) voire une « (mini) dissertation générale » (Calais et Doucet, 1987, p. 148), tandis que " l'essai littéraire [...] est une discussion sur un problème littéraire » (Pagès et Pagès-Pindon, 1982, p. 189). Ces solidarités entre ces deux genres proches ont des effets sur l'un et l'autre et produisent une forme de contamination des exercices : puisque la discussion se modèle en grande partie sur la dissertation (et notamment au niveau compositionnel), et qu'essai et discussion sont liés, le modèle dissertatif devient en quelque sorte le point commun des deux exercices. Le genre scolaire de l'essai s'éloigne ainsi davantage du genre littéraire dont il porte le nom, mais emprunte à la discussion sa dimension argumentative.

Le mouvement ne fait ensuite que s'amplifier : en 1983, la discussion est redéfinie par le $B O$ explicitement comme une production de texte argumentatif, avec comme critère d'évaluation «l'efficacité de l'argumentation » (alors que la définition de 1972 se contentait de souligner l'importance, pour tous les sujets, de 1'aptitude des candidats à « présenter leurs idées avec ordre »); cette même année, la dissertation générale (sous le nom de «composition française », cf. supra) vient remplacer l'essai. Les manuels de méthodes, qui commencent à fleurir à la fin des années 1980, rattachent désormais tous la discussion et la dissertation à l'argumentation, qui s'autonomise et devient un enjeu important des programmes. Pour Eterstein et Lesot par exemple (1990, p. 182), la dissertation est « un discours argumentatif $»$. La plupart des ouvrages soulignent explicitement les liens entre les deux exercices; certains même ne les séparent pas, et proposent une méthodologie commune (Crépin et al., 1988).

Dans cette logique de solidarité des exercices discussion/dissertation, la dissertation pouvait apparaître comme une version longue et/ou plus complexe (réservée aux classes de lycée, par exemple) de la discussion, écrit argumentatif plus souple et moins élitiste. Au milieu des années 1990, la « composition française sur un sujet général » et la discussion semblent donc former les deux facettes d'un

(6) Je reprends ici brièvement et partiellement des analyses développées dans Denizot, 2012 sur la scolarisation de l'argumentation.

(7) Ces dénominations des exercices ne sont pas innocentes, et Chervel (2006, p. $705 s q$.) a bien montré, à propos du passage des «exercices de style » à la « rédaction » à partir de 1880 dans l'école primaire, l'importance qu'elles peuvent avoir. 
même genre argumentatif scolaire que Darras et al. (1994), dans un ouvrage à destination des enseignants, choisissent d'ailleurs, de manière un brin provocatrice et paradoxale, de nommer « dissertation » :

Le choix de parler de la dissertation n'est pas neutre : le terme a disparu des Instructions officielles, et il n'existe pas, aux examens de l'Éducation nationale, d'exercice ainsi baptisé. Pourquoi alors le choisir comme le titre d'un ouvrage à caractère pédagogique? Parce que sous des appellations différentes se retrouve un même type d'écrits possédant des caractéristiques identiques et obéissant au même mode de production. [...] C'est donc un choix délibéré que de rassembler l'ensemble de ces exercices sous un même nom, afin d'envisager un apprentissage de la dissertation qui ne soit pas morcelé : il y a tout à gagner à montrer à l'élève au cours de sa scolarité que sous des appellations différentes, les exercices sont sensiblement les mêmes, comme, par conséquent, les capacités requises pour les réussir. ${ }^{(8)}$

Cela dit, cette recatégorisation de la dissertation en genre argumentatif n'allait pas sans difficulté. Comme le rappelait dès 1990 Michel Charolles (p. 10 ; c'est lui qui souligne) : «La dissertation, contrairement aux formes d'argumentation que l'on vient de citer, $n$ 'est pas tournée vers l'action: elle ne vise pas, au moins directement, à influer sur une décision. " Dans ce même numéro de Pratiques, Isabelle Delcambre (1990, p. 71) soulignait quant à elle que, sur un plan énonciatif et discursif, la dissertation relève davantage de la justification que de l'argumentation : «Il ne s'agit pas de convaincre ou persuader qui que ce soit [...], mais bien de développer sur ce sujet un discours acceptable par qui que ce soit ». Ce statut particulier de la dissertation a souvent été analysé, en particulier dans Pratiques (notamment 1990 ; cf. aussi Le Goff, 2009), et je n'y reviens pas. Il a fait l'objet de nombreuses propositions didactiques qui permettaient de prendre en compte sa complexité argumentative (régime discursif et énonciation, mise en scène de discours, etc.) ( $c f$. par exemple Delcambre et Darras, 1992 ; Darras et al., 1994 ; Delforce, 1992 ; etc.). Mais la dissertation y retrouvait une forme de légitimité, puisque son apprentissage était au service de l'apprentissage de l'argumentation et même, plus largement, « à situer dans la problématique plus générale d'une didactique des textes et des discours » (Delcambre et Darras, 1992, p. 17). C'est dans ce cadre qu'elle a pu retrouver un temps la faveur des didacticiens : «La dissertation quand même », comme l'écrivait Michel Charolles (1992) en titre de l'article qui ouvrait le numéro de Pratiques entièrement consacré à la dissertation. Sans enthousiasme donc, puisque « l'exercice est à l'évidence inadapté » mais avec le souci «d'en démonter minutieusement les rouages et donc de préparer méthodiquement les élèves à sa pratique »(p. 16).

\section{Un genre argumentatif ?}

La réforme de 1994, qui redonne à la dissertation son nom, semble à première vue s'inscrire dans ce mouvement de reconfiguration argumentative du genre : si la courte définition du $B O$ de 1994 ( $n^{\circ} 10$ du 28 juillet) n'évoque pas l'argumentation, le $B O$ de $1996\left(n^{\circ} 4\right.$ du 25 janvier) qui apporte des précisions sur les épreuves spécifie bien, dès la première phrase, qu' « il importe que [la dissertation] fasse appel à une argumentation » et rappelle ensuite l'importance de la problématisation. En 2001, les programmes relient la dissertation aux objets d'étude argumentatifs de la classe de première, notamment l'essai et la délibération, tandis que les nouvelles épreuves de l'EAF font de la dissertation le seul sujet de ce type, en suppri-

(8) Je respecte les choix typographiques des auteurs. 
mant la «discussion » : là encore, le texte officiel définit l'exercice comme une argumentation, élaborée à partir d'une problématique $\left(B O \mathrm{n}^{\circ} 26 \mathrm{du} 28\right.$ juin 2001 ${ }^{\left({ }^{(9)}\right.}$. Et ces deux impératifs - argumentation et problématisation — semblent désormais incontournables, comme le montre l'enquête par questionnaires de François Le Goff (2009) auprès de 82 enseignants : " pour une large majorité des répondants, 1'exercice de la dissertation entre dans le champ des pratiques argumentatives »; et plus des trois quarts d'entre eux déclarent que la réflexion problématisée est préférable à l'exposé de savoirs (p. 117-118).

Pourtant, l'exercice est redevenu depuis 1994 une dissertation littéraire, recentrée sur les savoirs littéraires et sur les objets d'études, et seule représentante au lycée de ce genre argumentatif scolaire qu'elle avait un temps formé avec la discussion. Or, si la question des contenus ne suffit pas à discréditer la dissertation comme genre argumentatif, elle remet cependant au cœur du genre une dimension qui était devenue secondaire quand le genre était à dominante argumentative, celle de la mise en scène des savoirs, de leur légitimité et de leur apprentissage. D'une certaine manière, la dissertation littéraire résiste à être décrite simplement comme un genre argumentatif, et la réduire à sa composante argumentative, loin d'être une aide à sa production, est sans doute un obstacle à la fois méthodologique et cognitif, tant elle reste aussi un genre évaluatif, dont la dimension thématique (celle des contenus) est centrale. Par ailleurs, elle n'est pas nécessairement le genre le plus à même de favoriser des apprentissages argumentatifs, et les propositions didactiques les plus intéressantes après 1994 sont généralement des propositions alternatives (voir par exemple celles de Caroline Masseron, 1997, autour notamment de l'écriture d'un traité d'argumentation).

D'ailleurs, les manuels actuels, s'ils proclament tous - en conformité avec les textes officiels - le caractère argumentatif de la dissertation, sont bien en peine de la rattacher au travail sur les genres argumentatifs, qui sont pourtant devenus des objets disciplinaires importants au lycée. Le caractère argumentatif de la dissertation s'affiche actuellement comme une composante essentielle du genre scolaire, mais n'est pas objet de travail ${ }^{(10)}$. Il y a même dans les manuels de méthodes une forme de régression par rapport aux années 1990 : le travail sur le « texte argumentatif » était alors corrélé à un travail assez systématique sur les arguments, les contre-arguments, la réfutation, etc., qui pouvait être une aide dans la production des écrits dissertatifs. Labouret et Meunier (1995) font ainsi de multiples renvois entre les chapitres concernant la dissertation et ceux concernant le texte argumentatif, les connecteurs, les types d'arguments et les stratégies d'argumentation, et même l'implicite - tous savoirs qui ont quasiment disparu des manuels de méthodes actuels, recentrés sur les objets d'étude et la littérature. La dimension argumentative de la dissertation se retrouve ainsi actuellement réduite à sa part la plus formelle et la plus « rhétorique », au sens le plus réducteur du terme. La version 2011 du manuel de Labouret propose ainsi une méthodologie en trois temps : « dégager la problématique », « chercher des arguments » et « organiser l'argumentation ». Si l'on excepte la référence à la problématique, qui est bien ici ce « simple rafraîchissement des exercices canoniques, comme la dissertation ou l'exposé »

(9) La définition de 2011, comme je le disais en début de cet article, est la même que celle de 2001 : cf. supra.

(10) De ce point de vue, il peut être rapproché de la " problématique », autre composante de la dissertation, injonction récurrente mais " point aveugle pour l'enseignant » (Nonnon, 2002) - et pour l'élève. 
qu'évoque Élisabeth Nonnon (2002, p. 38), les conseils sont minimalistes (« Il ne faut pas confondre arguments et exemples ») et d'un formalisme plutôt daté(11) : «On prépare l'enchaînement des arguments en prévoyant pour chacun d'eux un développement en trois temps : on exprime une idée ; on l'illustre ou on la justifie par l'analyse d'un exemple ; on conclut l'analyse de l'exemple en revenant au fil conducteur de l'argumentation » (p. 355).

Il me semble que l'impasse à laquelle on arrive, et qui fait de la dissertation, selon les témoignages de tous les correcteurs, un des sujets les moins choisis au baccalauréat, tient à cette difficulté actuelle de la saisir en tant que genre. La dissertation est en réalité, comme tous les genres, travaillée par différents composants qui, comme le souligne Canvat (1999, p. 86-87) à propos des genres littéraires (mais cela me semble transférable ici), ne sont pas toujours tous présents en même temps, mais qui peuvent éventuellement se décrire en termes de "dominante »: composants institutionnels, énonciatifs, fonctionnels, formels et thématiques. Si l'on considère le genre scolaire, c'est-à-dire le genre propre à l'école (en tant que sphère d'activité sociale), les composantes institutionnelles et fonctionnelles (évaluation, mise en scène de savoirs, etc.) sont essentielles, et dominent actuellement la composante argumentative, puisque la dissertation n'est pas (n'est plus?) prioritairement un exercice visant à évaluer des compétences et des savoirs argumentatifs. Autrement dit, les objectifs du genre « discursif» (scolaire) ne sont pas ceux affichés par la définition scolaire du genre « textuel» (pour reprendre Adam, 2012, qui distingue ainsi les «genres discursifs ou formes d'action discursives » et les «genres textuels ou formes dominantes de mise en textes (proto)typées ») ${ }^{(12)}$. La dissertation qui se présente comme un genre textuel argumentatif ne l'est que très indirectement : si argumentativité il y a, celle-ci se situe à un autre niveau que celle affichée, et a comme objectif de convaincre le correcteur que l'on sait quelque chose - et non pas de le convaincre d'une quelconque thèse à défendre. L'absence de véritable enjeu argumentatif, l'importance énorme prise par les aspects compositionnels et les caractéristiques formelles ainsi que par les composants thématiques, brouillent le genre et empêchent d'en comprendre le fonctionnement, les intérêts éventuels, et les limites.

Il est au contraire essentiel, pour clarifier les enjeux didactiques de la dissertation (et, peut-être, lui redonner une nouvelle vie), de la saisir à la fois dans son épaisseur historique, avec ses composantes génériques complexes qui témoignent de la « sédimentation» (Schneuwly, 2007) de l'objet, et à la fois dans ses relations avec les exercices qui l'accompagnent aux différentes époques, et avec lesquels se jouent des phénomènes de contamination ${ }^{(13)}$ et de reconfiguration. (Re)définir la dissertation comme genre nécessite ainsi de prendre au sérieux les tensions entre le feuilletage de l'héritage et les finalités disciplinaires qu'on lui assigne.

(11) Formalisme qui n'est pas sans rappeler le «paragraphe argumentatif » qui a eu un certain succès dans les années 1980-1990, et dont Isabelle Delcambre (1995) démontra (magistralement) les apories, dans un article qui n'a pas pris une ride.

(12) On retrouve ici un paradoxe présent dans un genre scolaire voisin, celui de l'écriture d'invention (Denizot, 2005), dont les objectifs affichés ne sont pas ceux réellement visés, et dont les sujets ont souvent « ce point commun de faire semblant de demander des productions non-scolaires » (p. 35).

(13) Phénomènes qui ne lui sont d'ailleurs pas spécifiques, comme le montre par exemple le cas de l'écriture d'invention, contaminée par la dissertation (Daunay, 2003 ; voir aussi Vibert, 2008). 


\section{Bibliographie}

ADAM, J.-M. (2012) : «Discursivité, généricité et textualité. Distinguer pour penser la complexité des faits de discours ", Recherches $n^{\circ} 56$, Les discours en classe de français, Lille, ARDPF, p. 9-27.

BÉNAC, H. (1949) : Vocabulaire de la dissertation, Paris, Hachette.

BENOIT, J.-P. (1999) : «L'émergence du discours et de l'argumentation dans l'enseignement du français », dans Benoit J.-P. (dir.), L'émergence du discours dans l'enseignement du français, Rennes, CRDP de Bretagne, p. 11-59.

BIET, C., BRIGHELli, J.-P., RisPail, J.-L. (1988) : Littérature 2. Techniques littéraires, Paris, Magnard.

BRUNEL, P. et COUTY, D. (collection) (1977) : Approches littéraires. 2. Les genres, Paris, Bordas.

Calais, É. et Doucet, R. (1987) : Organibac. Français I. Méthodes, Paris, Magnard.

CANVAT, K. (1999) : Enseigner la littérature par les genres, Bruxelles, De Boeck-Duculot.

Charolles, M. (1990) : «La dissertation quand même », Pratiques $\mathrm{n}^{\circ}$ 68, Metz, CRESEF, p. 5-16.

ChARTIER, A.-M. et HEBRARD, J. (1989/2000) : Discours sur la lecture. 1880-2000, Paris, BPI/Fayard.

CHERVEL, A. (2006) : Histoire de l'enseignement du français du XVII au XX siècle, Paris, Retz.

ClouARD, H. (1961) : La Composition française préparée (nouvelle édition revue et augmentée), Paris, Didier.

Crepin, F., Loridon, M., Pouzalgues-Damon, E. (1988) : Français. Méthodes et techniques, Paris, Nathan.

Darras, F., Daunay, B., Delcambre, I., VAnseVeren, M.-P. (1994) : Apprentissages de la dissertation $3^{e} / 2^{e}$, Lille, CRDP.

DAUNAY, B. (2003) : «Les liens entre écriture d'invention et écriture métatextuelle dans l'histoire de la discipline », Enjeux $n^{\circ} 57$, Littérature et écriture d'invention, Namur, CEDOCEF, p. 9-24.

DELCAMBRE, I. (1990) : «De l'argumentation à la dissertation. Analyse d'une démarche d'apprentissage ", Pratiques $n^{\circ}$ 68, La dissertation, Metz, CRESEF, p. 6988 .

- (1995) : «Faire écrire des paragraphes argumentatifs : une impasse didactique », Recherches $n^{\circ} 23$, Écrire d'abord, Lille, AFEF, p. 7-63.

DelCAMBRE, I. et DARRAS, F. (1992) : «Des modules d'apprentissage du genre dissertatif », Pratiques $n^{\circ}$ 75, Apprendre à rédiger, Metz, CRESEF, p. 17-43.

DELFORCE, B. (1992) : «La dissertation et la recherche des idées ou : le retour de l'inventio », Pratiques n ${ }^{\circ} 75$, Apprendre à rédiger, Metz, CRESEF, 1992, p. 3-16.

DENIZOT, N. (2005) : «L'écriture d'invention : écriture des genres ou nouveau genre scolaire? », Pratiques, ${ }^{\circ}$ 127-128, L'écriture d'invention, CRESEF, p. 31-44. - (2012) : « La scolarisation de l'argumentation dans l'enseignement secondaire. Analyse des textes officiels depuis $1970 »$, Recherches $\mathrm{n}^{\circ}$ 56, Les discours en classe de français, Lille, ARDPF, p. 135-148.

DENIZOT, N. et MABILON-BONFILS, B. (2012) : «La dissertation : déclinaisons disciplinaires d'un objet scolaire », Recherches en Didactique, Activités disciplinaires dans le secondaire, Villeneuve d'Ascq, REDLCT, p. 11-27.

Des GRAnges, C.-M. et MAGUelonne, M. (1929) : La composition française aux 
divers examens. Enseignement secondaire, enseignement primaire supérieur, Paris, Hatier.

ÉtersteIn, C., LESOT, A. (1990) : Le français au bac, Paris, Hatier.

GARCIA-DEBANC, C. (2001) : «L'enseignement de l'argumentation au lycée : entre approche linguistique et héritage rhétorique », Pratiques $n^{\circ} 111-12$, Metz, CRESEF, p.189-208.

GÉRAY, C. (1981) : 1981. Bac français (collection Profil formation annales), Paris, Hatier.

Glaudes, P. et Louette, J.-F. (1999) : L'essai, Paris, Hachette.

HOUdART-MEROT, V. (1998) : La culture littéraire au lycée depuis 1880, Paris/Rennes, ADAPT-PUR.

JEY, M. (1998) : La littérature au lycée: Invention d'une discipline (1880-1925), Recherches textuelles $\mathrm{n}^{\circ}$ 3, Metz, Université de Metz.

JuneauX, M. (1960) : Bac $1^{\text {ère }}$ partie. Sessions de 1959. Épreuves et corrigés, Paris, Bordas.

LABouret, D. et Meunier, A. (1995) : Les méthodes du français au lycée, Paris, Bordas.

LABOUReT, D. (dir.) (2011) : Méthodes et pratiques, Paris, Bordas.

LE GOFF, F. (2009) : " Enquête sur un écrit de savoir au lycée : la dissertation littéraire ", Pratiques n 143/144, Écrits de savoir, Metz, CRESEF, p. 111-126.

MACÉ, M. (2006) : Le temps de l'essai. Histoire d'un genre en France au XX $X^{e}$ siècle, Paris, Belin.

MASSERON, C. (1997) : "Pour une didactique de l'argumentation (écrite) : problèmes, objets et propositions ", Pratiques n 96, Enseigner l'argumentation, Metz, CRESEF, p. 7-61.

MORNET, D. (1934) : Cours pratique de composition française, Paris, Larousse.

NONNON, E. (2002) : «Formulation de problématiques et mouvements de problématisation dans les textes réflexifs : un point aveugle pour l'enseignant?», Spirale $\mathrm{n}^{\circ} 29$, Lire-écrire dans le supérieur, Lille, ARRED, p. 29-74.

PAGÈS, A. et PAGÈS-PIndon, J. (1982) : Le français au lycée. Manuel des études françaises, Paris, Nathan.

RINCÉ, D. (1980) : XIX siècle. Textes français et histoire littéraire. Classes des lycées, Paris, Nathan.

SCHNEUWLY, B. (2007) : « Genres écrits et oraux et forme scolaire. Enseignement et apprentissage de la langue première à l'école », dans Boré C. (dir.), Construire et exploiter des corpus de genres scolaires, Namur, Presses universitaires de Namur, p. 13-26.

THORAVAL, J. (1959) : La dissertation française au baccalauréat. 1. Auteurs et genres littéraires. Classes de Seconde et Première ( $7^{\mathrm{e}}$ édition), Paris, Armand Colin.

TOINET, J. (1947) : Dissertations littéraires et explications de textes (huitième édition augmentée), Paris, éditions de l'École.

VECK, B. (dir.) (1994) : La culture littéraire au lycée : des humanités aux méthodes?, Paris, INRP.

VIGNER, G. (1990) : «Argumenter et disserter : parcours d'une écriture », Pratiques $\mathrm{n}^{\circ}$ 68, La dissertation, Metz, CRESEF, p. 17-56.

VIBERT, A. (2008) : "Écriture d'invention et argumentation », dans Jean-François Massol (dir.), Écriture d'invention et ateliers d'écriture. Réflexions et pratiques didactiques pour aujourd'hui, Grenoble : Université Stendhal Grenoble 3, p.35-87. 